\title{
Philosophiques
}

\section{Reconnaissance et justice éducative}

\section{Thomas Michiels}

Volume 43, numéro 1, printemps 2016

URI : https://id.erudit.org/iderudit/1036469ar

DOI : https://doi.org/10.7202/1036469ar

Aller au sommaire du numéro

Éditeur(s)

Société de philosophie du Québec

ISSN

0316-2923 (imprimé)

1492-1391 (numérique)

Découvrir la revue

Citer cet article

Michiels, T. (2016). Reconnaissance et justice éducative. Philosophiques, 43(1), 93-113. https://doi.org/10.7202/1036469ar

\section{Résumé de l'article}

Si les théories de la reconnaissance ont permis de renouveler plusieurs domaines de l'éthique, peu d'auteurs se sont intéressés jusqu'à présent à leur apport aux débats éducatifs. Or, que peut précisément le concept de reconnaissance pour l'éthique de l'éducation ? C'est la question posée par cet article. Examinant de prime abord plusieurs propositions faites par la littérature, cette étude leur oppose ensuite une vision plus exigeante de la reconnaissance, soit, le fondement légitime d'une théorie relationnelle de la justice éducative.
Ce document est protégé par la loi sur le droit d'auteur. L'utilisation des services d'Érudit (y compris la reproduction) est assujettie à sa politique d'utilisation que vous pouvez consulter en ligne.

https://apropos.erudit.org/fr/usagers/politique-dutilisation/ 


\title{
Reconnaissance et justice éducative
}

\author{
THOMAS MICHIELS
}

Université Catholique de Louvain

thomas.c.michiels@uclouvain.be

\begin{abstract}
RÉSUMÉ. - Si les théories de la reconnaissance ont permis de renouveler plusieurs domaines de l'éthique, peu d'auteurs se sont intéressés jusqu'à présent à leur apport aux débats éducatifs. Or, que peut précisément le concept de reconnaissance pour l'éthique de l'éducation? C'est la question posée par cet article. Examinant de prime abord plusieurs propositions faites par la littérature, cette étude leur oppose ensuite une vision plus exigeante de la reconnaissance, soit, le fondement légitime d'une théorie relationnelle de la justice éducative.
\end{abstract}

ABSTRACT. - If the theories of recognition have allowed to regenerate numerous fields of ethics, few authors have so far shown an interest to their input in educational debates. Now what exactly can bring the concept of recognition to education ethics? This is the question raised by this paper. While initially examining several interpretations suggested in this litterature, this article further argues for a more demanding vision of the recognition: a legitimate and valuable basis of a relational theory of justice in education.

L'évolution de la littérature philosophique a ceci d'intéressant que celle-ci manifeste, depuis vingt ans au moins, un regain d'intérêt notable pour les questions éducatives ${ }^{1}$. Ce qui était il y a peu l'objet quasi-exclusif des sciences de l'éducation redevient source de curiosité pour les philosophes. Ainsi, l'autorité, la sanction, le rôle parental, ou les finalités éducatives constituent autant de sujets saisis par eux ${ }^{2}$. Cela est plus vrai encore des réflexions sur la justice éducative, très présentes sur ce terrain, qui s'inspirent à n'en pas douter des théories contemporaines de la justice sociale. Les œuvres de Rawls, Sen et Walzer, pour ne citer qu'elles, irriguent le propos de ceux qui évaluent actuellement la légitimité de normes, pratiques ou structures éducatives ${ }^{3}$. Depuis quelques années, un nombre encore très limité d'auteurs cherchent pourtant à approfondir ces débats à l'aune d'un autre cadre philosophique: celui des théories de la reconnaissance. Le statut de ces théories est certes différent, étant donné qu'elles ne forment pas clai-

1. L'auteur remercie Hervé Pourtois, Yolande Borremans ainsi que les membres du GIRSEF (Groupe interdisciplinaire de recherche sur la socialisation, l'éducation et la formation) pour leurs précieux retours sur les versions antérieures de ce texte. Cet article n'engage évidemment que son auteur.

2. Voir notamment et sans souci d'exhaustivité: Blais, Ottavi, Gauchet (2002), Renaut (2002), Avanzini et Mougniotte, (20I2), Allen, Reich (20I3), Swift, Brighouse (20I4).

3. On pensera par exemple ici à Michel Seymour et Alain Renaut qui usent tous deux du principe rawlsien de juste égalité des chances afin de critiquer l'iniquité actuelle des frais universitaires, au Québec comme en France (Seymour, 20I3; Renaut, 2007). 
rement ou exclusivement un paradigme de justice ${ }^{4}$. Il reste que le concept de reconnaissance ne serait pas dénué d'attraits pour penser l'éthique de l'éducation, et la justice éducative en particulier.

C'est cette prétention que nous aimerions clarifier dans cet article. Cet objectif nous invitera d'abord à proposer un bref examen de la littérature sur la question (I). Nous y évaluerons en l'occurrence diverses contributions: celle de Roger Monjo qui fait de la reconnaissance la base d'une critique de l'obligation scolaire; celle de Rauno Huttunen et Mark Murphy, qui voit dans ce concept un fondement normatif de la pédagogie radicale; ou encore, celle d'un auteur comme François Dubet qui centre l'intérêt de cette éthique sur sa capacité à enrichir nos descriptions de la justice et de l'injustice à l'école. Leur analyse sera l'occasion pour nous de découvrir et d'interroger une hypothèse rejetée par ces auteurs, à savoir que le concept de reconnaissance puisse constituer le noyau normatif d'une authentique théorie de la justice. C'est cette interprétation que nous tenterons ensuite de soutenir dans cet article, en identifiant le critère, l'objet ainsi que les exigences normatives possibles de cette approche (2). Nous défendrons qu'en spécifiant certains enjeux relationnels la reconnaissance permet d'approfondir une vision distributive de la justice et de concourir à ce titre à une visée d'égale liberté. Une ligne argumentative dont nous dégagerons finalement plusieurs conséquences en éducation (3).

\section{Regard critique sur la littérature}

Parce que tirer des concepts philosophiques vers un champ d'étude appliqué n'est pas chose aisée, permettons-nous d'amorcer notre réflexion par un renvoi critique aux auteurs qui nous ont précédé sur cette voie. Ceux-là ont le mérite incontestable d'avoir esquissé en pionniers plusieurs liens possibles entre reconnaissance et éducation.

Pour ces commentateurs, l'attrait principal du concept de reconnaissance réside dans l'anthropologie relationnelle qu'il sous-tend. Mettre l'accent sur le besoin, le désir et les conflits de reconnaissance qui animent l'humanité, c'est d'abord refuser la vision étroite de l'être humain qui apparaît dans les discours néo-libéraux : l'individu rationnel, le culte de la performance et du mérite, le rejet d'une culture de l'assistance (Monjo, 20I3, p. 45-46). Parler de l'homme à travers le concept de reconnaissance, c'est voir au contraire en celui-ci un être vulnérable, dont le développement dépend de son environnement relationnel et des formes de reconnaissance qu'il y forme. Ce modèle aurait l'avantage de nous sensibiliser à la sollicitude, au respect ou à l'estime que les individus cherchent à obtenir dans les

4. À l'exception de Fraser (2005), l'articulation entre reconnaissance et justice reste ambiguë chez des auteurs comme Honneth (2002) ou Taylor (I985). La reconnaissance ouvre en outre à une variété d'exigences normatives, comme l'appel aux dialogues interculturels, à l'amour ou à la solidarité, qu'est loin d'épuiser l'idée de justice. 
différentes sphères de leur vie, en nous montrant les effets potentiels de ces relations sur les possibilités de réalisation des individus. Il nous rendrait attentif aux effets moraux, et non plus seulement utilitaires, que des normes, politiques, structures, habitudes, ou pratiques peuvent produire sur des individualités. Il aurait la vertu de nous rappeler que c'est avant tout dans le langage moral de la reconnaissance et du mépris que nous ressaisissons des expériences comme celles de l'injustice. Voici esquissé en quelques mots le cadre qui va constituer pour ces commentateurs le point de départ de leur démarche appliquée.

Certains en feront le fondement normatif de dispositifs ou de pratiques pédagogiques. C'est le cas de Roger Monjo, Rauno Huttunen et Mark Murphy. D'autres encore y verront un langage moral aux vertus descriptives nous permettant d'approcher en éducation divers processus et expériences vécues. Un usage que l'on retrouvera notamment chez François Dubet ${ }^{5}$. Précisons brièvement ces deux pistes interprétatives pour en proposer ensuite une critique.

La première voie considère que cette anthropologie relationnelle ouvre à une éthique de la société aux implications éducatives originales. Elle revient à dire que la reconnaissance promeut ce qu'ils appellent une société décente: à savoir, une organisation sociale qui permettrait à tous ses membres de réaliser ses conceptions de la vie bonne, en les soutenant et en contribuant au développement de relations réussies, appliquées à eux-mêmes (Huttunen, Murphy, 20I2, p. I49). Dit encore autrement, cette société aurait pour vertu de reconnaître "le désir partagé par tous de mener une existence suffisamment digne pour permettre le maintien de l'estime de soi » (Monjo, 20I3, p. 53). Comme nous l'avons suggéré, cette exigence morale envers la société va, chez ces trois auteurs, s'articuler à des enjeux éducatifs précis. Aussi Roger Monjo va-t-il en faire le fer de lance de sa critique de l'obligation scolaire, là où Rauno Huttunen et Mark Murphy y verront un préalable nécessaire de la pédagogie radicale.

Pour le premier, il y aurait un paradoxe certain à vouloir souscrire à l'idée de société décente, tout en assumant une vision policière de l'obligation scolaire. Parce que cette dernière s'impose aujourd'hui en France comme une mesure légale assortie de sanctions, elle ne serait plus perçue par les élèves autrement que comme une contrainte qui s'applique à eux de l'extérieur. Si nous voulons qu'une société manifeste du respect pour ses membres en reconnaissant leur autonomie, nous devrions pour ce faire transformer cette obligation en un droit moral: celui de recevoir une éducation, comme nous recevrions une allocation universelle, garantie à vie. En abandonnant le registre de la sanction et du devoir juridique, estime Monjo,

5. Pensons aussi à une auteure comme Sally Power qui appréhende l'histoire des politiques éducatives anglaises à partir des trois paradigmes de la justice sociale selon Fraser redistribution, reconnaissance et représentation (Power, 20I 2). 
on ne produira pas une déscolarisation massive, mais on contribuera au contraire à réconcilier les acteurs sociaux avec l'école.

À côté de cette perspective plus conséquentialiste, Huttunen et Murphy verront de leur côté la société décente comme une condition de la pédagogie radicale. Par pédagogie radicale, Huttunen et Murphy entendent essentiellement se référer à l'éducation émancipatrice développée par Paulo Freire ${ }^{6}$. Sans jamais contester la valeur de cette dernière, ils vont en réalité questionner ses fondements éthiques. Car si Freire a donné une forme emblématique à cette pédagogie radicale, il aurait par contre insuffisamment contribué à sa fondation normative. S'agissant par exemple d'un cercle d'étude, il importe que dans cette pratique éducative les adultes puissent "apprendre à travailler, parler et s'écouter ensemble ", ils doivent pouvoir prendre les autres en considération et mettre en lumière les relations de pouvoir qui se cachent potentiellement dans leur situation d'apprentissage (Huttunen, Suoranta, 2006, p. Io). C'est là qu'intervient en l'occurrence l'idée de société décente. Dans sa pratique comme dans sa théorisation, la pédagogie radicale nécessite que des conditions recognitives soient remplies. Nos auteurs se basent sur les travaux d'Honneth (et par extension de Fraser) pour en identifier deux: la reconnaissance culturelle et la reconnaissance critique $^{7}$. La première ouvre à la reconnaissance pour laquelle les individus luttent au quotidien dans leurs différentes sphères sociales. Elle se centre sur le respect, l'amour et la sollicitude que l'on cherche à obtenir en éducation comme dans d'autres pratiques (Huttunen, Suoranta, 2006, p. 4). La seconde nous introduit à un niveau macro-politique: c'est-à-dire au besoin d'une justice politique et économique (Huttunen, Suoranta, 2006, p. 4), et à son influence morale sur la perception que les acteurs ont d'eux-mêmes. Voici mise à jour une double condition à «l'utopie» freirienne.

Celle-ci exprime une première façon d'user du concept de reconnaissance en éducation. Si la démarche de ces auteurs est originale et constitue par exemple un apport à la pédagogie de Freire, on regrettera néanmoins qu'elle ne parvienne à fournir une vision plus exhaustive et rigoureuse de ce

6. Il s'agit en résumé de l'appel à une pratique éducative "conscientisante" organisée autour du dialogue, de la découverte et du partage d'un monde commun, plutôt que sur des savoirs fixes à transmettre, réciter ou mémoriser par cœur. La pédagogie de Freire refuse l'opposition classique entre le maître savant et les élèves ignorants - la vision bancaire de l'éducation - pour lui préférer un cadre dialectique où prime la mise en recherche collective - l'acte cognitif -, l'égalité et le respect des personnes (voir Freire, I977 et 2013).

7. Précisons sur ce point que l'article de Huttunen et Murphy semble réemployer quelques paragraphes d'une réflexion menée antérieurement (Huttunen et Suoranta, 2006). Le problème est que cette double orientation se rapporte alors à Honneth (reconnaissance culturelle) et Fraser (synthèse de la reconnaissance culturelle et critique), tandis que l'article de $20 \mathrm{I} 2$ semble exclusivement rattacher ces orientations aux sphères de reconnaissance honnethiennes. L'ambiguité nous paraît dommageable car on ne voit pas très bien, aux vues de la posture moniste de Honneth $(2000,2003)$, comment ce dernier pourrait pleinement souscrire à cette «reconnaissance critique». À tout le moins, l'article de $20 \mathrm{I} 2$ ne l'indique pas. 
que la reconnaissance peut en propre pour l'éthique de l'éducation. Ne pourrait-elle forger un regard singulier sur la forme des programmes, structures et pratiques scolaires, pédagogies et systèmes d'évaluation? Au-delà de l'obligation scolaire, ces exigences morales ne justifieraient-elles pas une déscolarisation de la société, comme le pensait Ivan Illich (I97I)? Avant de nous saisir modestement de ces problèmes, nous souhaitons aborder le dernier usage «éducatif » qui est fait de la reconnaissance. Un usage plus sociologique qui, en marge des fondations normatives, se borne à une description des sentiments d'injustice. Cette valeur relative de la reconnaissance, on la retrouve chez François Dubet.

Suivant ce dernier, le principal attrait de la reconnaissance réside dans sa «force descriptive» (Dubet, 2008, p. I48). Elle nous permettrait de rendre compte du contexte subjectif dans lequel se vit et s'énonce éventuellement un sentiment d'injustice. Car on doit ici concéder à Honneth d'avoir vu que les injustices sont actuellement éprouvées «comme une atteinte à son identité, à sa propre estime, à sa capacité d'agir et de se sentir pleinement membre d'une société» (Dubet, 2008, p. I44). Le besoin de reconnaissance possèderait ainsi le mérite sociologique d'englober «la plupart des plaintes » (Dubet, 2008, p. I47). Voilà ce que l'on doit aux théories de la reconnaissance; voici leur attrait descriptif, que Dubet ne manquera pas d'observer dans ses analyses du milieu scolaire. Il faut selon lui arrêter de se focaliser sur les seules performances des élèves et sur les systèmes d'évaluation, "comme si l'unique fonction de l'école était de classer les élèves " (Dubet, 2009 , p. 43). Il faut que la sociologie s'enquière aussi de la face subjective des injustices scolaires. Ce changement d'orientation permet à Dubet d'affirmer que le mépris éprouvé par les élèves renvoie en fait à une pluralité de principes de justice: l'égalité, le mérite et l'autonomie. Les élèves se considèrent comme étant fondamentalement égaux et attendent qu'on les traite avec respect en dépit de la hiérarchie scolaire. Pareillement, ils se demandent aussi dans quelle mesure les évaluations, notes, appréciations et examens sont justes et objectifs, et, ce faisant, si on évalue réellement leur mérite (Dubet, 2009, p. 45). Enfin, ils sont attachés à leur autonomie, à ce qui leur confère une valeur propre, une singularité, un esprit critique, à ce qui les rend maîtres de leur propre vie (Dubet, 2009, p. 48). Le sociologue s'intéressera aux situations qui laissent apparaître des tensions entre ces principes. Plus globalement, il manifestera un souci récurrent pour les effets moraux de la méritocratie scolaire. S'il s'agit selon lui d'une fiction nécessaire, elle n'en est pas moins cruelle, actuellement, envers les vaincus du système, et ce, parce qu'elle ne les protège pas suffisamment du "mépris des vainqueurs " (Dubet, 2004, p. 3I).

Plus que dans les travaux de Monjo, Huttunen et Murphy, c'est chez Dubet que la reconnaissance se spécifie le plus en une authentique éthique de l'éducation. Lorsque les premiers plaident en faveur d'un droit à l'éducation ou d'une pédagogie radicale, leur idée de la reconnaissance reste fonciè- 
rement générale, et on comprend assez peu comment elle se décline en concepts éducatifs. Condition sociétale ou fruit d'une suppression de l'obligation scolaire... il s'agit finalement d'en appeler à une "société décente" dont on ne saisit jamais parfaitement les contours. À l'inverse, Dubet montre que la reconnaissance fournit un accès privilégié aux expériences morales des acteurs. Une entreprise qu'il a su appliquer au monde du travail, mais également aux écoles en montrant toute la singularité des formes du mépris dans ce milieu. Son apport reste pourtant modéré par le refus d'interpréter la reconnaissance comme le sol d'une approche plus normative de la justice. Face subjective des injustices, la reconnaissance semble chaque fois se dérober à elle-même pour nous ouvrir à une pluralité d'exigences de justice. Ce qu'il lui manque, c'est un noyau normatif qui lui est propre, car "non seulement l'expérience de la reconnaissance n'a pas d'unité, mais elle repose sur des principes antagoniques» (Dubet, 2008, p. I 54). Ce dont elle semble également dépourvue, c'est d'un critère de justice permettant de distinguer ceux qui ont des souffrances, besoins et demandes légitimes, de ceux qui ne les ont pas. Une expérience peut bien faire souffrir et affecter négativement mon bien-être moral, mais cela n'en fait pas nécessairement une situation injuste qui appellerait réparation (Dubet, 2008, p. I44-I 45). Aussi la portée de la reconnaissance devrait-elle se limiter à la seule description des expériences subjectives de l'injustice. Ces situations légitimeraient au mieux la compassion ou la fraternité (Dubet, 2008, p. I 59), et en matière scolaire, une attention notable envers la violence de la sélection et la hiérarchisation des filières ou réseaux, mais il serait dommageable d'étendre plus loin le mépris vécu.

Tout en concédant volontiers que l'institution scolaire soit au carrefour d'une pluralité de modèles de justice, et que la reconnaissance soit un langage moral qui puisse nous renvoyer à eux, nous voudrions pourtant contester l'idée que celle-ci n'ait absolument pas de noyau normatif propre, d'unité ou de critère de justice. Certes, lorsque nous parlons de reconnaissance, nous pouvons bien viser des objets comme le mérite, l'égalité ou l'autonomie - les trois sphères de reconnaissance décrites par Honneth nous y amènent d'une façon ou d'une autre. Nous tenterons cependant de défendre que cela n'enlève rien à l'unité conceptuelle de cette théorie: soit, que la justice recognitive porte sur des enjeux chaque fois relationnels et qu'elle puisse les rassembler et évaluer à l'aune d'un critère d'égale liberté, en produisant finalement des normes propres.

\section{Vers une théorie recognitive de la justice}

\section{Reconnaître pour libérer}

Concédons d'abord que la reconnaissance ne soit pas immédiatement un problème politique de justice sociale. Il est vrai que nous émettons et recevons chaque jour des appréciations morales. Notre vie quotidienne est remplie de 
ces expériences tantôt heureuses, tantôt malheureuses: l'attention d'un ami envers nos besoins, les félicitations d'un instituteur pour notre effort scolaire, etc. Les théoriciens de la reconnaissance n'ont pas l'ambition démesurée de réguler ces expériences de la vie quotidienne, encore moins de forcer la reconnaissance sous toutes ses formes et dans tous ses lieux. L'attention d'Honneth envers la reconnaissance idéologique en est une preuve patente: il faut parfois aussi s'en méfier (Honneth, 2006a, p. 245-274). Dès lors, si la reconnaissance n'est pas juste en toute circonstance, elle ne le devient vraisemblablement que lorsqu'elle réhabilite les possibilités égales de réaliser sa liberté. Dit encore autrement, la souffrance liée au déni de reconnaissance devient injuste lorsqu'elle affecte ces mêmes possibilités. Voici la thèse que nous nous proposons de soutenir, en sachant qu'elle n'épuise pas la normativité disponible au sein des théories de la reconnaissance. Axel Honneth, Nancy ou Charles Taylor utilisent des termes distincts lorsqu'ils approchent ce critère: ils parlent tour à tour de parité participative à la vie sociale, d'émancipation, de traitements égaux, de chances égales de se réaliser ou encore de liberté sociale, etc. Il est clair également que chacun organise spécifiquement ses modalités. Un objectif commun les unit néanmoins: affirmer que le mépris devient à un moment injuste, c'est-à-dire lorsqu'il diminue ou réduit les possibilités d'action égales des acteurs, leur égale liberté.

Qu'appelle-t-on ici égale liberté? Dans une acception formelle et minimale d'abord, il est patent que ces théories participent à l'idéal politique du libéralisme classique en reprenant à leur compte la défense juridique des libertés fondamentales. Les individus doivent acquérir de manière égale un statut qui leur assure des droits de choisir et de réaliser librement leur conception de la vie bonne. Ce qui implique la protection égale de droits individuels (liberté physique, vie privée, mariage, etc.), politiques (liberté de vote, de réunion...) et socio-économiques (liberté de travailler, d'être éduqué....). Lorsque Fraser prend "l'égalité juridique» comme condition d'une parité participative ou de lutte contre "l'exclusion politique», lorsque Honneth conçoit une seconde forme de mépris en termes de "privation de droits ", lorsque Taylor enfin avalise un modèle de société libérale garante des «droits fondamentaux», on retrouve bien cette conception formelle de la liberté. Mais l'attention au mépris et à la reconnaissance se situe plus spécifiquement autour de la réalisation de la liberté réelle: c'est-à-dire sur l'ensemble des capacités, moyens, chances ou ressources que nécessite la concrétisation de ces droits par leurs détenteurs. Emmanuel Renault l'a d'ailleurs bien compris ${ }^{8}$. Rawls et Sen avaient déjà manifesté un intérêt pour

8. Avant qu'Honneth ne déplace son propos de l'intégrité psychique vers la liberté sociale, Renault avait déjà bien cerné le fait que la reconnaissance constitue une "condition de l'usage de la liberté ». Ce dernier défend que le concept de reconnaissance se rattache non seulement aux droits formels, mais aussi aux droits sociaux, aux «conditions de la liberté » ainsi qu'à nos conditions de vie (Renault, 2004, p. 65). 
cette réalité proprement morale et son incidence sur la vie bonne. Pour Rawls, c'est un bien social premier qui doit faire l'objet d'une distribution équitable; pour Sen, qui fera un pas de plus, c'est un facteur qui peut affecter l'accès et la conversion de multiples ressources - et donc un problème plus général, plus diffus. Honneth, Taylor et Fraser accompliront et systématiseront au fond cette intuition. La reconnaissance n'accomplit pas l'exigence d'égale liberté en s'imposant comme un droit absolu. Le concept indique seulement que l'exercice de notre liberté réelle est conditionné par des formes de reconnaissance réciproques, que celles-ci affectent aussi et en partie nos possibilités d'accéder et de s'approprier des ressources. Aussi est-ce dans le prolongement des œuvres de Rawls et de Sen qu'il faut localiser la justice recognitive. Fraser le reconnaît explicitement lorsqu'elle inscrit sa théorie de la justice dans l'espace évaluatif des capabilités9. La chose nous paraît également justifiable chez Honneth, pour qui la notion de liberté est enrichie par l'idée hégélienne de "vie éthique ». Cela signifie que la liberté n'est pas dissociable des formes sociales d'existence, qu'elle ne peut se découvrir et se réaliser comme telle qu'au sein des pratiques et institutions sociales. La liberté quitte ainsi l'abstraction du droit pour s'auto-réaliser dans différents contextes de vie (famille, marché, État) traversés par des enjeux de reconnaissance (Honneth, 2008, p. 89). Cette perspective est directement reprise par Honneth pour qui la norme de liberté «réelle» (Honneth, 2008, p. 86) ne peut être indifférente aux conditions sociales de son émergence et, ce faisant, aux structures de la reconnaissance. En effet, selon lui, la liberté implique non seulement une protection juridique, mais en outre et surtout, le fait de prendre part à la coopération sociale, à des réseaux de communication, aux différentes sphères de la vie sociale. Et si c'est par ce biais que le concept déploie sa pleine portée, il est dès lors nécessairement conditionné par les relations de reconnaissance inséparables de ces institutions et pratiques: la sollicitude dans la sphère des relations intimes et privées; le respect dans la sphère publique; l'estime dans la sphère professionnelle et associative. Il en résulte que les relations de reconnaissance ne recouvrent pas seulement des enjeux identitaires et psychologiques, mais participent bien désormais à un enjeu libéral.

Ainsi, la reconnaissance peut bien renvoyer au besoin d'amour, à certaines demandes d'égalité, d'autonomie ou à la considération de son mérite. Si la justice recognitive revêt néanmoins une certaine consistance normative, c'est parce qu'elle permet de rassembler ces exigences autour d'une même

9. "Bohman is also mistaken in treating my framework as antithetical to the capability model of justice. Thanks to some recent exchanges with Ingrid Robeyns and Kevin Olson, I have come to see that the principle of participatory parity operates in 'the evaluative space of capabilities', as Amartya Sen would say, because it assesses social arrangements in terms of the degree to which they assure people the capability to participate fully, as peers, in social life. Contra Bohman, then, my framework belongs to the family of capability approaches " (Fraser, 2007, p. 319). 
réalité relationnelle et de cette visée libérale. L'argument paraîtrait purement rhétorique s'il n'avait pas autant d'incidence sur la façon de saisir des objets et de suggérer des remèdes au sein de cette théorie. Si ce qu'il y a de notable dans une attente morale de reconnaissance était par exemple son strict renvoi à une exigence égalitariste, on ne comprendrait pas que la justice recognitive puisse donner autant de valeur au sentiment de mépris qui y est associé. Or, si la description du mépris importe, c'est parce que celui-ci est susceptible d'imposer un mode d'action qui ne résume pas dans ce cas à la seule norme d'égalité. De la même façon, ce qui caractérise en propre la justice recognitive dans son rapport aux normes - on le verra - c'est également son souci pour les discriminations qu'elles présentent sur la base de marqueurs culturels et symboliques. On pourrait par exemple contester l'objectivité d'un critère méritocratique, présent dans une école, qui ne chercherait pas à lutter contre l'effet des contingences sociales initiales dans le parcours des élèves - donc sur une base distributive. Mais nous dirions que ce qui anime la justice recognitive, dans cet exemple, c'est la possibilité que le mérite soit apprécié différemment selon les traits culturels ou symboliques appartenant à ces élèves. Nous reviendrons sur ces aspects dans la section suivante.

Enfin, si la justice recognitive participe à cette tradition libérale, elle suggère selon nous un usage pourtant différent de la reconnaissance. Celle-ci ne s'impose pas comme une obligation redistributive: il ne peut être question de distribuer la reconnaissance de manière égale et inconditionnelle comme un bien parmi d'autres, ou d'assurer, comme c'est le cas chez Rawls, quelque chose comme des bases sociales au respect de soi. Selon Feriel Kandil, il s'agirait plutôt d'envisager la reconnaissance comme un acte de réparation: un acte dont le caractère contraignant ne se présenterait à nous que lorsqu'il s'agit de "réparer les préjudices liés à la reconnaissance négative» (Kandil, 20I2, p. 2I9), lorsque le mépris est tel qu'il affecte foncièrement la façon d'accéder à ces ressources et/ou de les utiliser. Le terme a le mérite d'inviter à une différenciation des registres. On peut simplement regretter son incapacité à intégrer les autres formes de responsabilité qu'implique ce paradigme, c'est-à-dire des actes préventifs du mépris, un traitement dialogique du mépris vécu, ou la promotion d'attitudes vectrices de reconnaissance. Comme nous le verrons plus loin, ces stratégies débordent d'une façon ou d'une autre l'acte purement réparateur. Aussi identifieronsnous ce cadre à une approche plutôt relationnelle de la justice sociale, c'està-dire, à un type d'analyse qui se soucie de ce qui, dans le tissu relationnel des sociétés, affecte négativement l'appropriation de ressources, possibilités ou capabilités constitutives d'une égale liberté. Par relationnel, nous renvoyons à une définition élargie de l'expression qui couvre non seulement des interactions informelles entre individus et groupes, mais aussi celles qui sont institutionnalisées. On peut donc parler de relations individuelles, sociales, juridiques, etc. À tout le moins, ce choix marque un changement de priorité 
théorique: de primaire, la distribution de biens devient ici un problème secondaire, médiatisé par les facteurs relationnels qui en affectent le destin. On dira donc quelque part que la reconnaissance n'est pas l'objet d'évaluation de ces théories; à travers elle, ce sont les conditions relationnelles d'exercice de l'égalité des libertés de base qui sont visées. Les évaluations morales négatives deviennent par conséquent une question de justice lorsqu'elles modifient en profondeur la manière dont des personnes engagées au sein de la coopération sociale peuvent se référer à leurs droits, désirs, aptitudes, ressources ou chances.

Ces considérations sur le critère d'égale liberté invitent sans nul doute à la modestie. En rabattant la normativité du concept de reconnaissance sur ce dernier, nous admettons faire dépendre ce paradigme de justice de celui de la redistribution. Les demandes de reconnaissance n'y sont en effet prises au sérieux que dans la mesure où elles mettent en péril l'appropriation de biens. Est-ce dès lors un modèle autonome de justice? Il serait plus juste de parler de dépendance. À tout le moins, nous voudrions défendre que cet "ajout» offre une certaine complémentarité aux lectures distributives de la justice - en évaluant comment les jugements moraux, mentalités ou discours idéologiques peuvent avoir un effet sur la distribution de biens.

\section{Analyser le mépris et le combattre}

Le soin que Taylor, Honneth et Fraser portent à l'égale liberté les encourage à décrire et évaluer ce qui, sur le plan moral, affecte négativement ce critère: les formes du mépris. Voici leurs objets. Il faut pouvoir les isoler, en montrer les symptômes, les relier à des attentes normatives ou à des structures institutionnelles, mais surtout en explorer l'incidence objective ou potentielle sur la liberté réelle des acteurs sociaux. Les théories de la reconnaissance présentent selon nous deux conceptions distinctes du mépris: comme réalité institutionnelle et comme réalité vécue.

La première formulation témoigne essentiellement du point de vue fraserien. Celle-ci estime qu'une approche réellement politique de la justice doit identifier une réalité objective, et ce faisant, ne sélectionner dans son analyse que les formes de mépris qui font l'objet d'une institutionnalisation. Suivant cette voie, il s'agit d'évaluer si une loi, une politique, une habitude ou règle de vie présente des aspects qui discriminent explicitement certains groupes d'individus en fonction d'un marqueur culturel ou symbolique (religion, genre, ethnie, langage, etc.). Constater par exemple qu'un conseil d'enseignants manifeste une habitude récurrente des hommes à interrompre plus souvent les femmes, à parler plus, plus longtemps, ou à ignorer largement les interventions des femmes (Fraser, 2005, p. I 20). Dans cette perspective, la mise en évidence du mépris ne repose pas sur des considérations psychologiques, dit-elle, mais sur la référence à un ensemble de valeurs objectivées, sur les normes que des pratiques et arènes institutionnalisent. 
La méthodologie de la seconde conception, inspirée de Taylor et Honneth, est fondée sur une analyse du mépris vécu. Le point focal se déplace: il ne porte pas d'emblée sur le caractère visiblement inique d'une norme institutionnalisée mais sur un éprouvé subjectif, sur l'expérience vécue du mépris. Ce qu'il faut rendre apparent, c'est ce qu'un individu expérimente et exprime, dans le langage articulé ou par des symptômes, comme étant des formes de mépris. Le mépris vécu apparaît à la conscience subjective au moyen d'affects négatifs - sentiments de honte, colère, humiliation, etc. - qui lui révèlent un décalage entre des attentes normatives et une situation de fait. Cela ne veut pas encore dire que l'individu soit aux prises avec une injustice objective. L'analyse doit d'abord identifier si ce sentiment est verbalisé ou observable à travers ses effets psycho-sociaux. Dans le premier cas, il s'agit d'évaluer la structure inique discriminée par le discours public des acteurs sociaux, et la pertinence de la critique. L'intérêt de placer le curseur sur le mépris vécu réside dans le fait de ne pas rejeter le second cas. Que se passe-t-il lorsque cette expérience négative n'entre pas dans le langage articulé et reste bornée à ses effets psycho-sociaux? La procédure devra interpréter elle-même la source potentielle de l'injustice, remonter à une pratique ou norme possiblement responsable. Si la réaction morale de l'individu s'avère finalement disproportionnée au terme de l'enquête, sans justifier par conséquent la nécessité de remédiations institutionnelles, l'analyse renferme toutefois l'avantage incontestable d'appréhender ces sentiments d'injustice. Car même illégitimes, ils peuvent produire des effets réels décrits en termes de pathologies, handicaps ou violences. Un sentiment d'exclusion, par exemple, peut affecter durablement la perception de soi et de ses aptitudes, limiter ses désirs, ses ambitions, ou la représentation de ses chances. Fictif, ce dernier produit pourtant des obstacles réels au critère d'égale liberté. S'il n'y a pas lieu de modifier une norme institutionnalisée dans ce contexte, on peut cependant justifier l'utilité d'actions qui permettent l'expression et la réflexion sur ce sentiment.

Parce qu'elles développent une approche relationnelle de la justice et se focalisent sur deux formes de mépris, les théories de la reconnaissance se dotent finalement de deux normes distinctes: objectiver le mépris vécu; réparer le mépris institutionnalisé. La première se rattache à ce que nous appellerons l'exigence de dialogues réflexifs. La seconde prend essentiellement la forme de stratégies pragmatiques nous renvoyant soit à des actions correctrices, soit à des actions transformatrices.

Selon Taylor et Honneth, lorsqu'un individu ou un groupe se sent victime du mépris, le risque existe qu'il se replie sur lui-même ou manifeste des comportements violents. Ce vécu n'assure pas l'objectivité du mépris, et encore moins la présence d'une injustice, rappelons-le, mais parce que ce risque potentiel bute contre le critère d'égale liberté, il faut pouvoir agir en conséquence. Taylor et Honneth donneront une forme dialogique à ce traitement du mépris vécu. Il faut que l'individu ou le groupe qui se sent méprisé 
puisse traduire ce sentiment dans une parole et que cette parole soit entendue. Qu'elle soit ensuite validée et qu'il ne fasse pas de doute qu'elle reflète une véritable atteinte à la justice n'est pas le problème immédiat. Ce qui importe par exemple pour Honneth est qu'il y ait expression verbale, que l'on puisse "conférer aux méprisés et aux exclus la force individuelle d'articuler leurs expériences dans l'espace démocratique» (Honneth, 2006b). La valeur du langage est ici de réfléchir l'expérience vécue du mépris. Par le langage, l'individu ou le groupe peut endosser les attitudes des autres et se ressaisir comme un objet. L'idée n'est pas de dire que le langage amène à la paix sociale et au consensus, mais simplement que l'expression verbale permet de sortir du ressentiment et de ses conséquences pathologiques potentielles. L'éthique du dialogue interculturel de Taylor précise à sa manière cette même exigence. Face à des conflits communautaires pour la reconnaissance, il est utile qu'un dialogue puisse se créer ou se maintenir. Avant tout jugement, ce dialogue a pour but de produire un "common mind», c'est-à-dire un espace de compréhension commune, un espace où l'on puisse exprimer et écouter le contenu des attentes, frustrations et demandes des acteurs impliqués. Il faut pouvoir comprendre le point de vue d'autrui et par conséquent être sensible "à ses émotions et aspirations, à ce qu'il trouve admirable et méprisable chez lui et les autres, à ce qu'il craint, etc.» (Taylor, I985, p. I I9 - notre traduction). La notion de dialogue réflexif nous semble réellement intéressante parce qu'elle introduit à une fonction curative du langage. Si cette dernière ne risque pas de révolutionner la psychologie sociale et individuelle, elle se distingue pourtant des fonctions couramment associées au langage par la philosophie politique contemporaine: soit, par exemple, de ses usages rhétoriques et stratégiques, soit de sa vision habermassienne centrée au contraire sur l'entente et le consensus argumentatif.

Il reste alors à traiter du second principe recognitif: la "réparation » du mépris institutionnalisé, l'action contre des normes iniques institutionnalisées. Cette stratégie renvoie au problème suivant: que fait-on lorsqu'on est parvenu à démontrer le caractère injuste d'une norme - c'est-à-dire, le fait qu'elle défavorise systématiquement un groupe en vertu d'un marqueur culturel ou symbolique? Une évidence commune aux trois auteurs consiste à dire que nous devrions laisser aux acteurs sociaux le soin de remédier à ce déni de reconnaissance. Le meilleur remède est encore celui qui résulte de discussions inclusives entre les personnes concernées. Fraser affirme que le remède au mépris institutionnalisé devrait témoigner d'une analyse pragmatique de la situation. Ainsi, une politique publique injuste réclame un changement de politique; une loi inique, un changement de loi, etc. (Fraser, 2005, p. 80). Lorsque ce qui est nié est l'appartenance à une humanité commune, le remède consiste en une reconnaissance universaliste; à l'inverse, le déni d'une particularité culturelle exige une reconnaissance de la différence (Fraser, 2005, p. 55). Il n'y a donc pas de solution a priori, l'analyse doit 
précéder la prise de décision. Allant plus loin, Fraser ajoute qu'on peut vouloir distinguer entre remèdes correcteurs et remèdes transformateurs. Les premiers s'apparentent à une action palliative, ils corrigent les "effets" d'une structure inique en laissant cette dernière intacte. Il s'agit par exemple de revaloriser un groupe d'individus méprisés par une réallocation superficielle du respect. Les seconds modifient les «causes profondes " de l'injustice en réorganisant radicalement la disposition d'une structure inique. Cela impliquera notamment de déconstruire ou de déstabiliser les différenciations existantes au sein de la structure culturelle d'une société (Fraser 2005, p. 36). Le choix entre ces deux stratégies reste évidemment aux mains des acteurs sociaux.

\section{Mépris et justice éducative}

Après ces considérations plus générales, il reste naturellement à interroger l'apport de cette théorie au monde éducatif. Cette application semble d'abord légitime du fait que la justice recognitive permet l'examen de différentes institutions, sans considération d'échelle ou de restriction à quelque chose comme une "structure de base". Il suffit pour s'en convaincre d'observer les espaces dans lesquels évoluent les œuvres des théoriciens de la reconnaissance. Parce qu'elle porte sur une réalité morale, la justice recognitive va de l'analyse des expériences privées et intimes jusqu'à celle de normes institutionnalisées. On peut donc dire qu'elle ne connaît pas de limites, si ce n'est évidemment par le choix de son objet propre qu'est le mépris.

Si la justice recognitive s'applique potentiellement au monde éducatif, on ajoutera de surcroît qu'elle y trouve un intérêt notable, et cela est d'autant plus vrai du milieu scolaire. Contrairement à d'autres institutions, l'école jouit comme la famille d'un statut spécial. Elle ouvre principalement ses portes à un public d'enfants et d'adolescents, public qu'elle introduit au "monde", qu'elle acculture, qu'elle socialise. Les rapports de reconnaissance qui s'y forment acquièrent ce faisant une haute signification, car ils sont premiers dans l'ordre du développement psychique des jeunes. Honneth ne dit-il pas que la sphère de l'amour et de l'amitié prime sur les autres sphères de reconnaissance, parce qu'elle leur est antérieure et se répercute sur leur destin? (Honneth, 2000, p. I3I). On peut par conséquent faire l'hypothèse que l'institution scolaire s'adresse à des jeunes qui n'ont a priori pas autant de résistance psychique qu'un adulte normal face à l'humiliation et au mépris. À tout le moins, il est certain que la justice recognitive trouve un attrait supérieur à l'école dès lors que celle-ci n'est pas seulement un lieu de socialisation, mais un lieu où s'acquièrent aussi des ressources et aptitudes utiles à l'épanouissement personnel, politique et professionnel des individus. Parce que le mépris peut affecter la manière dont un jeune s'approprie des ressources éducatives, il affecte par conséquent les conditions de réalisation de sa liberté. Et l'effet de ce critère de justice est profond puisqu'il engage en grande partie le développement futur de ce jeune. 
Cela étant dit, la justice recognitive nous semble dès lors capable d'identifier trois responsabilités vis-à-vis des institutions éducatives.

I) La première dérive immédiatement de l'appel à lutter contre le mépris institutionnalisé. Ce qui s'impose à ces dernières, c'est le devoir d'éviter que leurs normes et pratiques n'institutionnalisent des modèles de valeur qui portent préjudice à certains de leurs membres, au seul motif qu'ils possèdent des traits culturels ou symboliques propres. Cette responsabilité couvre une multitude de situations allant, par exemple à l'école, des conditions d'accès et de réussite des écoliers, aux procédures de recrutement des enseignants, en passant par les pratiques d'enseignement, etc. La justice recognitive demande que ces normes et pratiques scolaires soient passées au crible et que l'on examine si elles affectent négativement les porteurs de certains marqueurs culturels ou symboliques. S'il s'avère que celles-ci réduisent visiblement les conditions de leur égale liberté, il est juste d'agir à leur encontre. L'originalité de cette première exigence est de porter une attention particulière non plus seulement aux structures scolaires, comme c'est le cas dans les lectures redistributives de la justice, mais aussi à la culture scolaire: c'est-à-dire aux mentalités, aux discours idéologiques, aux distinctions et désavantages qu'ils génèrent.

L'échec scolaire, par exemple, est un phénomène fréquemment associé à des enjeux redistributifs dans les débats contemporains. On l'articule communément à des questions de financement du système scolaire ou d'allocation équitable des ressources éducatives. En revanche, peu courants sont les propos qui le relient à l'iniquité de nos représentations sociales. Le jugement d'échec et sa fréquence peuvent-ils néanmoins prétendre à une pure objectivité ou neutralité? À suivre plusieurs sociologues et associations, le jugement d'échec ne se contenterait pas de rétribuer l'élève selon son "mérite ", mais participerait au processus par lequel une école cherche à affirmer son caractère élitiste. $\mathrm{Ce}$ jugement interviendrait également dans l'effort des enseignants pour recueillir l'approbation de leurs collègues. L'échec varierait potentiellement d'ailleurs en fonction du genre des élèves et relèverait de modèles culturels d'évaluation centrés sur la nécessité de classer, de hiérarchiser au sein d'une classe (selon une courbe de Gauss par exemple). Enfin, ce jugement s'accompagnerait parfois d'appréciations morales critiquables, comme un pronostic sur les capacités futures d'un élève ou sur la nature de son intelligence (une possession fixe dont nous sommes pourvus ou non $)^{10}$. Dans ce contexte, les

10. Sur ces perspectives, voir notamment: G. M. Walton, "The Myth of Intelligence ", in D. Allen, R. Reich (eds.), Education, Justice and Democracy, Chicago, London, The University of Chicago Press, 20I3, p. I55-I72; M. Crahay, Peut-on lutter contre l'échec scolaire? Bruxelles, Editions de Boeck, 2003 ; B. Galand, "Réussite scolaire et estime de soi », in Sciences humaines, $\mathrm{n}^{\circ} 5$ spécial "L'école en questions", 2006; Ligue des Droits de l'enfant Asbl, «L'échec scolaire est une maltraitance », in Journal de la Ligue des Droits de l'enfant, J.-P. Coenen (dir.), dossier enseignement $\mathrm{n}^{\circ} 3$, juillet-août-septembre 20I0; J. Cornet, «Il faut maintenir le redoublement! », in Traces de changements, $\mathrm{n}^{\circ} 22 \mathrm{I}$, mai-juin $20 \mathrm{I} 5$, p. 4-5. 
marqueurs de bons et mauvais élèves, d'élèves capables et méritants ou de cancres ont-ils encore une légitimité ? Comment un écolier peut-il encore facilement «croire en sa capacité d'apprendre alors qu'il vient d'être mis en échec et qu'il entend dire autour de lui que l'intelligence est un don?" (Crahay, 2003, p. 255). Connaissant les effets néfastes potentiels de l'échec-redoublement sur le développement des élèves, on peut ici se demander si les représentations culturelles dont procèdent ce genre de distinctions ne devraient pas être déconstruites, parce qu'elles manifestent des formes de mépris institutionnalisé qui portent atteinte au critère d'égale liberté. L'institution scolaire avantagerait ou désavantagerait ici certains élèves en fonction de stéréotypes et discriminations liés au genre, mais aussi et plus largement selon les marqueurs symboliques qu'elle véhicule et leur appliquerait précocement en préjugeant de leur «mérite", de leur intelligence et de leurs possibilités futures. La justice recognitive ne rejette pas absolument les logiques de différenciation. La reconnaissance sociale et professionnelle d'Honneth en témoigne. Elle dénonce pourtant ici une catégorisation partiale et presque immédiate qui semble peu soucieuse des rythmes d'apprentissage différents ou des habitudes inégales devant les exigences scolaires. Elle rejette un jugement qui déborde de la copie aux capacités de l'élève, et de l'individu présent à l'individu futur.

Cette culture d'échec ne représente au fond qu'une illustration possible de ce à quoi la justice recognitive rend sensible. On admettrait volontiers qu'en deçà des pratiques pédagogiques l'administration du mépris social puisse également être attachée à l'organisation politique du système scolaire. Nous pensons par exemple, dans un contexte belge francophone, à la la régulation de l'offre scolaire qui prend une dimension de "quasimarché». Cette expression désigne le fait d'une concurrence entre écoles pour attirer les élèves, dans une situation de libre choix d'un établissement par les familles, et d'un financement proportionnel au nombre d'élèves inscrits (Dupriez et Dumay, 20II, p. 84). Cela génère une volonté de diversifier l'offre pour se rendre plus attractif que l'établissement voisin, et progressivement, une hiérarchisation du système scolaire. À son sommet apparaissent les écoles, filières et options socialement valorisées, un enseignement général, souvent élitiste, et un public sur-représenté par les milieux socio-économiquement favorisés. À sa base résident au contraire les écoles et filières dites de relégation, un enseignement surtout technique et professionnel, et un public essentiellement défavorisé. Le quasi-marché offre dans ce contexte un appui structurel aux logiques de dualisation et d'homogénéisation, voire de sélection précoce des publics scolaires. L'iniquité de cette logique tient au fait qu'elle renforce structurellement l'effet de l'origine sociale dans les parcours (ce que le pouvoir politique reconnaît d'ailleurs explicitement ${ }^{11}$ ). Elle relève en outre d'un usage faisant qu'elle

11. Gouvernement de la Fédération Wallonie-Bruxelles, Pacte pour un enseignement d'excellence. [En ligne: http://www.enseignement.be/index.php? page=25703\&ne_id=22 I0], publié en janvier 2015 (consulté le 29 janvier 20I5). 
autorise des évaluations: ne plus servir les acquis des jeunes et découvrir leurs difficultés, mais viser leur seule orientation précoce dans des hiérarchies d'excellence. Chaque échelon de cette pyramide offrant des possibilités éducatives et des formes de reconnaissance inégales, on associera également ce quasi-marché à une forme possible de mépris institutionnalisé. Si ce modèle organisationnel s'enracine dans l'histoire de la Belgique francophone, et singulièrement dans les conflits entre laïcs et catholiques, on est en droit de se demander si on ne pourrait pas lui trouver des solutions de rechange plus justes. À titre illustratif, revaloriser l'enseignement technique, notamment, en en faisant un choix légitime plutôt qu'une voie de garage (action correctrice); ou encore accroître le pilotage du système scolaire par le politique, de sorte que s'atténuent progressivement les différences perçues entre établissements (action transformatrice).

2) À côté de cette première responsabilité, la justice recognitive réclame également l'institutionnalisation d'espaces de dialogues réflexifs. Cette revendication fait suite au problème du mépris vécu et à la nécessité de l'objectiver dans le langage. Dans le contexte scolaire, ce devoir couvre un large panel d'expériences et de personnes. Il porte par exemple sur la figure de l'enseignant et l'impression qu'il peut avoir que son travail est dénigré par une partie de la population, ainsi que par certains parents. Il peut tout aussi bien concerner des étudiants d'origine étrangère et leur sentiment d'être méprisés (exclusion, invisibilité) par un instituteur utilisant régulièrement et exclusivement des prénoms français dans la construction de ses exemples. La question du mépris vécu s'applique à tous les acteurs de l'éducation et cela, pourvu que les interactions entre ces derniers s'accompagnent d'attentes déçues de reconnaissance. La contrainte est de faire exister des lieux capables de réfléchir ces sentiments d'injustice, non seulement pour atténuer ces émotions négatives et les dangers liés à leur incorporation, mais également pour servir de base à l'action si leur expression permet de découvrir une norme réellement inique. Ce que nous appelons en réalité «institutionnalisation» ne préconfigure aucunement la forme de ces espaces de dialogue: il n'est pas nécessairement question ici d'une structure rigide, ni même unique. En fonction du problème et de sa gravité, on envisagera ainsi différentes stratégies dans le contexte scolaire. Ces dialogues peuvent prendre la forme d'entretiens individuels avec un psychologue scolaire, d'une discussion informelle animée par un enseignant, de rencontres entre parents et enseignants, ou entre représentants politiques et enseignants, etc. La justice recognitive dit surtout ici l'importance qu'il y ait quelque chose plutôt que rien, et que ce quelque chose prenne une forme dialogique. En s'inspirant de l'éthique du dialogue interculturel développée par Taylor (I994), on perçoit l'intérêt d'une norme d'inclusion et d'un effort d'intercompréhension qui précède tout jugement. Suivant Honneth, ajoutons qu'il faut ici éviter les pratiques générant une fausse reconnaissance ou une reconnaissance idéologique (Honneth, 2006a). Appliquée au milieu scolaire, cette exigence trouve une justification particulière dans les travaux de Valérie 
Caillet. Face aux élèves qui se disent victimes d'une injustice, ou face aux manifestations de ce sentiment négatif, la sociologue suggère de " déconstruire le plus tôt possible ces perceptions et [...] d'établir un dialogue avec les élèves, et ce aussi bien au sein de la classe que tout au long de l'année dans le cadre d'un travail collectif» (Caillet, 2006, p. 69). Approfondissant son idée, elle ajoute qu'on peut notamment «veiller à la création d'espaces de parole permanents favorisant l'écoute entre enseignants et élèves et où les jugements scolaires ne seraient plus en jeu, en présence d'acteurs aux éventuels conflits» (Caillet, 2006, p. 69). Une argumentation scolaire qui s'inscrit dans la droite lignée de la justice recognitive.

Cet impératif dialogique est-il foncièrement original en éducation? N'est-il pas déjà effectif dans un grand nombre de nos systèmes scolaires? On peut le penser, à regarder ne serait-ce que les programmes de prévention et de lutte contre la violence, le harcèlement ou le décrochage présents dans le monde occidental, qui accordent une valeur certaine aux dispositifs de médiation, aux contacts accrus entre les familles et les écoles, ou encore à l'intégration du vécu personnel des élèves au sein des cours. L'appel à saisir ces enjeux dans une perspective communautaire et dialogique est en outre une tendance récurrente des rapports internationaux en la matière ${ }^{12}$. La justice recognitive conduit ainsi à une seconde responsabilité dont l'attrait en éducation est par conséquent limité actuellement. On dirait avec Rawls que celle-ci offre surtout l'occasion d'une réconciliation avec nos politiques publiques. Elle reste néanmoins une exigence qu'il n'est pas futile de garder à l'esprit et de pousser peut-être davantage en avant. Les attentats terroristes de 2014 et 2015 en ont quelque part témoigné ${ }^{13}$. De sécuritaire, le défi a rapidement été perçu comme étant scolaire (notamment en France et en Belgique), et il a alors fallu identifier les façons d'y répondre. À côté des cours de citoyenneté ou d'enseignement moral et civique, la proposition a pu être lancée de donner une place accrue au vécu subjectif des élèves, d'encourager notamment des pratiques proches des Conseils présents dans la pédagogie institutionnelle ${ }^{14}$. Que l'on y adhère ou non, ce genre d'invocation suggère à tout le moins que notre principe recognitif n'est pas encore pleinement effectif, et qu'il constitue une balise normative qui peut encore faire sens.

12. Voir à titre illustratif: Conseil de l'Europe (20II). Combattre la violence à l'école. Rapport final de la réunion. [En ligne: http://www.coe.int/t/dg $3 /$ children/violence/OsloReport_ fr.pdf] (consulté le 22 août 20I 5). UNESCO (2009), En finir avec la violence à l'école: guide à l'intention des enseignants. [En ligne: http://unesdoc.unesco.org/images/o0 I 8/00 I 84 I/I 84 I 62f. pdf] (consulté le I7 février 2015).

13. Nous pensons par exemple à la tuerie au Musée juif de Bruxelles le 24 mai 20I4, aux attentats de janvier 20I 5 à Paris qui ont visé Charlie Hebdo et le magasin Hyper Cacher, ou encore à ceux plus récents du $\mathrm{I} 3$ novembre $20 \mathrm{I} 5$.

14. À titre illustratif, voir Dubois, Robbes et Jospin (2OI 5 ). Ceux-ci défendent l'institutionnalisation de lieux de parole et d'écoute «où les interrogations et les difficultés culturelles et d'appréhension sociale des élèves puissent être entendues et prises en compte ». 
3) Outre la lutte contre le mépris vécu et institutionnalisé, la justice recognitive offre un appui à une dernière responsabilité: celle qui consiste à promouvoir des relations de reconnaissance réussies. Il s'agit moins ici d'une exigence contraignante que d'un devoir moral au sens large du terme. Ce que la justice recognitive peut vouloir encourager en matière scolaire, ce sont les attitudes et règles de vie commune qui renforcent la confiance, le respect et l'estime de soi. Elle s'intéresse dans cette perspective à tous les facteurs ayant un effet moral potentiel sur l'intelligence affective des individus - leur capacité à identifier et exprimer un ressenti ou un besoin -, leur aptitude à se considérer comme autonome et à prendre la parole en public, ainsi que leur propension à produire et développer leurs compétences. Ces notions ouvrent à une éthique de l'éducation relativement ouverte qui concerne l'ensemble des acteurs du milieu scolaire. À considérer ce triple objet, on réalise d'ailleurs la portée de cette éthique, en ce qui concerne tant les pratiques pédagogiques que la liberté laissée aux enseignants, la vie quotidienne à l'école, etc. S'il s'agit de promouvoir les facteurs favorables de la confiance, du respect et de l'estime de soi, cet objectif ne possède pas la force contraignante des propos qui précèdent. On ne peut en son nom imposer par exemple des relations d'amitié entre écoliers, ou demander à un enseignant d'apprécier également des travaux très inégaux. Elle offre seulement un regain de justification à certaines stratégies spécifiques. Telle est la critique faite par Monjo de l'obligation scolaire, qui dérive selon nous de cette troisième responsabilité. Plus généralement, une école qui base explicitement ses relations internes sur le respect mutuel et la politesse participe en un sens à ce but moral. Il en va de même des pratiques pédagogiques qui s'efforcent d'atténuer la violence que la sélection scolaire et la rétribution du mérite peuvent générer envers les perdants du système. Cette invitation donne aussi du poids aux actions qui cherchent à prévenir l'apparition du mépris et des sentiments d'injustice. On ajouterait à cette triple exigence (confiance, respect, estime de soi) que les écoles soient aussi invitées à ne pas se focaliser sur un seul axe de reconnaissance, à l'exclusion des autres. Dans cette perspective, il s'agirait par exemple de ne pas rétribuer les seules tâches scolaires qui mettent les élèves en contact avec leur utilité sociale présente et à venir, mais de valoriser également celles qui leur permettent d'exprimer sans jugement leurs besoins et émotions, ou celles qui fondent leur autonomie critique. En réalité, l'intérêt pour cette troisième exigence nous semble surtout résider dans le regard complémentaire qu'elle permet de porter sur certains débats houleux en éducation: que ce soit par exemple sur la pertinence des systèmes de notations et classements scolaires, ou sur celle que certains associent à une libéralisation accrue des parcours scolaires ${ }^{15}$. À côté de l'efficacité ou de l'équité, cette norme recognitive se

15. Par cette notion de «libéralisation", nous désignons les tentatives visant à diminuer la contrainte exercée par l'institution scolaire dans sa forme traditionnelle. C'est-à-dire: à 
présenterait comme un autre critère donnant du poids aux analyses visant par exemple à estimer la tension engendrée par les logiques scolaires très compétitives, ou à celles qui évaluent plus globalement l'effet du cadre de vie scolaire sur la confiance en soi des élèves ${ }^{16}$.

Ces quelques pistes témoignent à notre sens de ce que la justice recognitive peut notamment pour l'éthique de l'éducation. Si on accepte la participation de la reconnaissance au critère d'égale liberté, on trouvera de prime abord une certaine légitimité à notre tentative. Ces trois principes ont en tout cas l'audace de dépasser une fonction exclusivement descriptive et d'indiquer comment l'idée de reconnaissance peut se décliner en concepts plus opératoires pour l'éducation. À l'instar de Fraser, nous pensons par ailleurs que cette approche de la justice renferme une valeur complémentaire, eu égard au paradigme de la redistribution. On voit qu'elle introduit à des enjeux relationnels originaux dont certains, comme la culture scolaire, nous paraissent plus difficilement accessibles à un cadre d'évaluation redistributif - et même à un cadre comme celui de la justice rawlsienne qui présente pourtant une "théorie» de la reconnaissance ${ }^{17}$. Inversement, on dirait tout autant que la justice recognitive ne peut se suffire à elle-même et qu'il serait par exemple tout à fait dommageable de réduire un phénomène comme l'échec scolaire à une stricte question de mépris.

\section{Bibliographie}

Allen, Danielle, et Rob Reich. Education, Justice and Democracy, Chicago, London, The University of Chicago Press, 2013.

Avanzini, Guy, et Alain Mougniotte. Penser la philosophie de l'éducation, Lyon, Éditions de la Chronique sociale, 20 I 2.

Blais, Marie-Claude, Dominique Ottavi et Marcel Gauchet. Pour une philosophie politique de l'éducation, Paris, Hachette Pluriel, 2002.

Caillet, Valérie. «Sentiment d'injustice et violence scolaire », in Revue de Recherches en éducation, $\mathrm{n}^{\circ} 37,2006$, p. 63-7I.

Cornet, Jacques. "Il faut maintenir le redoublement!", in Traces de changements, $\mathrm{n}^{\circ} 22 \mathrm{I}$, mai-juin $20 \mathrm{I} 5$, p. 4-5.

rendre notamment les cours facultatifs, à diminuer le temps passé à l'école ou encore à alléger la formation commune en multipliant les possibilités éducatives individuelles. Si ces propositions sont issues en grande partie des pédagogies dites libertaires, on les retrouve actuellement dans les critiques adaptatives de l'institution scolaire.

16. Nous ne faisons ici qu'offrir un traitement introductif à ces problématiques. À suivre certains résultats de la recherche en éducation, on dirait entre autres que la justice recognitive semble plus largement réalisée par des logiques d'évaluation qui prennent place dans un climat d'entraide et de bienveillance, et où les élèves sont moins invités à entrer en compétition les uns avec les autres qu'avec eux-mêmes (Vianin, 2006). La justice recognitive donnerait l'avantage aux évaluations formatives, ancrées dans la pédagogie différenciée, qui permettraient de retarder et d'adoucir le caractère sélectif des hiérarchies scolaires d'excellence (Perrenoud, I998).

17. Voir Lazzeri, 2004; Seymour, 2008. 
Crahay, Marcel. Peut-on lutter contre l'échec scolaire? Bruxelles, Éditions de Boeck, 2003.

Dubet, François. L'école des chances. Qu'est-ce qu'une école juste? Paris, Seuil, 2004 .

—. "Injustice et reconnaissance », in Revue Esprit, $\mathrm{n}^{\circ}$ 7, 2008.

—. "Conflits de justice à l'école et au-delà », in M. Duru-Bella, D. Meuret (dir.) Les sentiments de justice à et sur l'école, Bruxelles, Éditions de Boeck, 2009.

Dubois, Arnaud, Olivier Jospin et Bruno Robbes. «Pour une véritable éducation sociale et citoyenne: renforcer et développer l'usage des heures de vie de classe », 2015 [http://www.meirieu.com/ECHANGES/heure_vie_de_classe. pdf] (consulté le I4 juillet 20I 5 ).

Dupriez, Vincent, et Xavier Dumay. "Les quasi-marchés scolaires: au bénéfice de qui ? ", in Revue française de pédagogie, $\mathrm{n}^{\circ} \mathrm{I} 76,20 \mathrm{I}$.

Fraser, Nancy. Qu'est-ce que la justice sociale: redistribution ou reconnaissance? Paris, Éditions La Découverte, 2005.

—. "Identity, Exclusion, and Critique: A Response to Four Critics », in European Journal of Political Theory, 6:305, 2007.

Freire, Paulo. Pédagogie des opprimés, Paris, Maspero, I977.

—. Pédagogie de l'autonomie, Toulouse, Éditions Érès, 2013.

Galand, Benoit. "Réussite scolaire et estime de soi », in Sciences Humaines, $\mathrm{n}^{\circ} 5$ spécial «L'école en questions », 2006.

Honneth, Axel. La lutte pour la reconnaissance, Paris, Éditions du Cerf, 2000.

—. "Reconnaissance et justice", in Le passant ordinaire, $\mathrm{n}^{\circ} 38,2002$.

—. La société du mépris. Vers une nouvelle théorie critique, Paris, Éditions La Découverte, 2006a.

- - "Sans la reconnaissance, l'individu ne peut se penser en sujet de sa propre vie», in Philosophie Magazine, $\mathrm{n}^{\circ}$ 5, 2006b.

—. Les pathologies de la liberté, Paris, Éditions La Découverte, 2008.

- Freedom's Right. The Social Foundations of Democratic Life, Cambridge, Polity Press, 20I4.

Honneth, Axel, et Nancy Fraser. Redistribution or Recognition? A Political-Philosophical Exchange, London/New-York, Verso, 2003.

Illich, Ivan. Une société sans école, Paris, Éditions du Seuil, I97I.

Kandil, Feriel. Fondements de la justice, Paris, Presses Universitaires de France, $20 \mathrm{I} 2$.

Lazzeri, Christian. «Le problème de la reconnaissance dans le libéralisme déontologique de John Rawls ", in Revue du MAUSS, $\mathrm{n}^{\circ}$ 23, 2004.

Ligue des Droits de l'Enfant ABSL. "L'échec scolaire est une maltraitance ", in J.-P. Coenen (dir.) Journal de la Ligue des Droits de l'Enfant, dossier enseignement $\mathrm{n}^{\circ} 3$, juillet-août-septembre 20 Iо.

Monjo, Roger. "Obligation scolaire et droit inconditionnel à l'éducation de base ", in Formation et profession, $\mathrm{n}^{\circ}$ 2I (3), 2013.

Perrenoud, Philippe. L'évaluation des élèves. De la fabrication de l'excellence à la régulation des apprentissages. Entre deux logiques, Bruxelles, Éditions de Boeck, I998.

Power, Sally. «Redistribution, reconnaissance et représentation: parcours de la lutte contre l'injustice sociale et des changements de politique éducative ", in $E d u$ cation et Sociétés, $\mathrm{n}^{\circ} 29,2012 / \mathrm{I}$. 
Rauno Huttunen, et Mark Murphy. «Discourse and Recognition as Normative Grounds for Radical Pedagogy: Habermasian and Honnethian Ethics in the Context of Education ", in Studies in Philosophy and Education, vol. 3 I, $\mathrm{n}^{\circ}$ 2, 2OI 2, p. I37-I 52 .

Rauno Huttunen et Juha Suoranta. "Critical and Cultural Orientation in Radical Adult Education ", in C. Torres, A. Antikainen and P. Harinen (eds.) From the Margins: Adult Education, Work and Civil Society, Amsterdam, Sense Publishers, 2006.

Renault, Emmanuel. L'expérience de l'injustice, Paris, Éditions La Découverte, 2004. Renaut, Alain. La libération des enfants. Contribution philosophique à une histoire de l'enfance, Paris, Bayard, 2002. Égalité et discrimination, Paris, Éditions du Seuil, 2007.

Seymour, Michel. De la tolérance à la reconnaissance, Montréal, Boréal, 2008.

—. Une idée de l'université. Propositions d'un professeur militant, Montréal, Boréal, 20I3.

Swift, Adam, et Harry Brighouse. Family Values: The Ethics of Parent-Child Relationships, Princeton, Princeton University Press, 20I4.

Taylor, Charles. "The Nature and Scope of Distributive Justice ", in Philosophy and the Human Sciences - Philosophical Papers 2, Cambridge, Cambridge University Press, I985.

—. Multiculturalisme, différence et démocratie, Paris, Flammarion, I994.

Vianin, Pierre. La motivation scolaire. Comment susciter le désir d'apprendre? Bruxelles, Éditions De Boeck, 2006. 